\title{
Anabases
}

ANABASES Traditions et réceptions de l'Antiquité

6 | 2007

Varia

\section{Maya BURGER et Claude CALAME (éd.), Comparer les comparatismes. Perspectives sur l'histoire et les sciences des religions}

Corinne Bonnet

\section{OpenEdition}

\section{Journals}

Édition électronique

URL : http://journals.openedition.org/anabases/3394

DOI : 10.4000/anabases.3394

ISSN : 2256-9421

Éditeur

E.R.A.S.M.E.

Édition imprimée

Date de publication : 1 octobre 2007

Pagination : 251-253

ISSN : 1774-4296

\section{Référence électronique}

Corinne Bonnet, « Maya burger et Claude CAlame (éd.), Comparer les comparatismes. Perspectives sur

I'histoire et les sciences des religions ", Anabases [En ligne], 6 | 2007, mis en ligne le 01 janvier 2012, consulté le 21 octobre 2019. URL : http://journals.openedition.org/anabases/3394 ; DOI : 10.4000/ anabases.3394

Ce document a été généré automatiquement le 21 octobre 2019

(c) Anabases 


\title{
Maya BURGER et Claude CALAME (éd.), Comparer les comparatismes. Perspectives sur l'histoire et les sciences des religions
}

\author{
Corinne Bonnet
}

\section{RÉFÉRENCE}

Maya BURGER et Claude CALAME (éd.), Comparer les comparatismes. Perspectives sur l'histoire et les sciences des religions, Paris-Milan, Edidit \& Archè, 2006, 238 p.

17 euros /ISBN 2-912770-06-8.

1 On considère souvent, à juste titre, que la méthode comparative est la plus représentative de l'histoire ou des sciences des religions; c'est, pour le dire avec N. Gasbarro, une "priorité structurale» (p.19). Le grand mérite de ce volume est de montrer qu'il n'existe pas une démarche comparative, mais bien des comparatismes qui se distinguent les uns des autres par leurs présupposés théoriques ou épistémologiques, comme par leurs modalités opératoires. Partant, en comparant les comparatismes, on peut assurément mieux comprendre le sens, la portée et la validité de ces méthodes.

Deux contributions introductives cernent les objectifs du volume. Dans cette section intitulée «Les méthodes comparatives en jeu: remarques introductives sur l'histoire des religions", Maya Burger et Claude Calame présentent les «démarches comparatives » de l'histoire et des sciences des religions, tandis qu'Yvan Bubloz étudie l'oscillation du comparatisme "entre tremendum et fascinans". On nous y rappelle, à bon escient, que le comparatisme naît dans l'alvéole des sciences du langage et de l'évolutionnisme, et dans le cadre d'une «réflexion critique partagée entre universalisme et relativisme" (p.6). Mis en danger de nos jours par les replis disciplinaires et par la frilosité épistémologique, qui se contente d'une histoire des 
disciplines comme lieu de réflexivité, le comparatisme doit se déployer avec plus de conviction dans le but, notamment, de réaffirmer et de préciser, en ces temps de regain d'intérêt pour le "religieux ", son positionnement propre qui ne coïncide nullement avec celui de la théologie des religions. Celle-ci, en effet, pratique un autre type de comparatisme, que l'on pourrait qualifier de «hiérarchique» et d'essentialiste. Fascinante, mais redoutable, la comparaison suscite encore la méfiance et le débat: doit-elle déboucher sur une meilleure connaissance des divers domaines de spécialisation ou plutôt conduire à la modélisation et à la théorisation "visant à une explication globale de la culture ou de la religion ", c'est-à-dire à l'émergence de "systèmes religieux " pris en compte dans leur dimension dynamique, donc historique, et proposés comme des modèles provisoires d'intelligibilité ? Y.Bubloz montre bien comment les divers courants, les diverses écoles se sont tour à tour engagés dans un comparatisme morphologique (donc phénoménologique) ou historique (donc singularisant), analogique ou généalogique. Les enjeux sont donc considérables et ils touchent au statut même de l'histoire des religions, traversée par des approches diverses, voire divergentes, pourtant toutes enracinées dans la méthode comparative.

Huit contributions proposent ensuite des dossiers, essentiellement thématiques, mais nourris d'une réflexion épistémologique et destinés à éclairer les modalités de mise en œuvre de la comparaison, ses limites, ses résultats. Huit « exercices de comparaison » qui construisent leur objet au confluent de l'analogie et de la différenciation.

N. Gasbarro étudie l'évolution de la pratique du comparatisme en histoire des religions depuis le structuralisme de Lévi-Strauss. Entre le risque de généralisation à visée universaliste et celui de l'histoire différentielle, la comparaison doit opérer sur les codes culturels (y compris les codes naturels élaborés par la pensée historique dans des contextes donnés), par rapport auxquels la religion peut fonctionner comme le code prioritaire, ou du moins avoir cette ambition. N. Gasbarro consacre des pages intéressantes à l'articulation, au sein de la religion, entre orthodoxie et orthopratique, ainsi qu'au concept d'idolâtrie comme clé de lecture de la religion des autres.

P. Bornet consacre son exercice de comparaison aux rabbis et brahmanes : partant de contextes éloignés, que va donner la comparaison portant sur le rapport entre l'hospitalité et la pratique des sacrifices? En domaine rabbinique, les stratégies d'hospitalité, pensées comme un rituel, peuvent dès lors répondre aux mêmes objectifs qu'un sacrifice, voire se substituer à lui. Pareillement, dans la tradition brahmanique, le repas d'hospitalité a valeur de sacrifice domestique solennel. La comparaison interculturelle ainsi instruite fait émerger un lien partagé entre hospitalité et sacrifice, donc une analogie formelle et fonctionnelle, mais aussi des nuances et des différences : «l'hospitalité ne se charge pas de la même portée et n'est pas associée au sacrifice pour les mêmes raisons » (p. 69).

6 Toute naturelle et indispensable qu'elle soit, la comparaison n'en est pas moins complexe, souligne ensuite A.W. Geertz. Elle suppose le recours à des typologies, à des catégorisations qui ne s'imposent pas de soi, car reposant sur une pensée hiérarchique implicite, elle-même fondé sur des théories de la nature, des hommes et du monde. Intéressantes les pages où l'A. enracine la pratique comparative dans les mécanismes cognitifs : « elle est partie intégrante de la pensée analytique » (p. 83). La prière sert ici de champ d'expérimentation, en convoquant notamment les théories modernes de la communication et de la sémiotique, mais sans perdre de vue la dimension sociale du phénomène. 
7 Y. Bubloz applique, pour sa part, une méthode comparative de type analogique ou homologique (et non généalogique) aux discours d'Augustin et de Porphyre sur le salut. Trois types de considérations sont objet du rapprochement entre vision chrétienne et vision néoplatonicienne: la condition humaine, entre passions et impassibilité, les causes de la souffrance, les moyens pour y remédier (grâce ou effort individuel). De cette très belle enquête ressort avec force et nuances la variabilité des réponses culturelles à un problème donné, dans l'espace et le temps.

8 Le mythe de Médée, dans sa dimension langagière ou plus exactement énonciative et discursive, est le terrain de comparaison différentielle choisi par U.Heidmann. Elle précise, avant d'entrer dans le vif de son analyse, avec beaucoup d'acuité, les principes épistémologiques de sa démarche. Trois «moments » et contextes discursifs différents sont ensuite analysés: Apollonios de Rhodes, Sénèque et Sylvia Plath, pour faire émerger les modalités de "mise en langue ", donc de sémantisation du mythe, en dehors de toute approche essentialiste qui attribuerait au mythe une "substance " universelle.

9 Autre modulation de la comparaison: les regards croisés, auxquels recourt M. Burger pour étudier le yoga global et, au-delà, les échanges d'idées et de pratiques entre l'Inde et l'Occident. Des synthèses créatives, "subtil mariage entre l'universel et l'unique " (p. 185) naissent de ce va-et-vient entre cultures indiennes et cultures européennes aux $\mathrm{XIX}^{\mathrm{e}}$ et $\mathrm{XX}^{\mathrm{e}}$ siècles.

10 Comparer l'Islam et le christianisme: E. Pace nous propose de se livrer à une expérience de laboratoire de type "néo-wébérien». Jésus et Mahomet sont donc analysés, d'un point de vue cette fois généalogique, quant à leur profil de " personnalité mobile et charismatique " suscitant la construction d'un système de croyance (avec fixation du contenu vrai du message délivré oralement au départ d'une capitalisation symbolique antérieure) et exerçant un pouvoir de communication, reposant notamment sur la vertu d'improvisation. C'est naturellement la dimension évolutive qui ressort de ce type de comparaison.

11 C. Calame propose une contribution finale sur «L'histoire comparée des religions et la construction d'objets différenciés : entre polythéisme gréco-romain et protestantisme allemand ». En décentrant son regard, en parcourant l'espace que les anthropologues balisent par les concepts d'" étique » et "émique », le spécialiste du polythéisme peut prendre conscience du fait que son objet ne correspond pas à une catégorie indigène. Sa focalisation change donc selon qu'on adopte un point de vue indigène ou celui du discours savant, forcément extérieur et étranger, mais néanmoins marqué par les paradigmes identitaires et culturels propres à l'analyste. C. Calame parcourt donc à la fois les modes de saisie savants du polythéisme grec, de Dumézil à Vernant, en passant par Lévi-Strauss et Detienne, et les clés de lecture antiques, les «manières grecques » de concevoir le polythéisme, notamment par le biais du puissant opérateur logique que sont les généalogies et les timai. Il introduit enfin le troisième élément de sa triangulation comparative : le regard protestant. La lecture de Troeltsch notamment nous introduit à une autre façon de penser la religion et Dieu, par le biais d'une démarche abstraite, fondamentalement philosophique et normative.

12 Le volume que nos collègues suisses ont édité est passionnant. La richesse de la réflexion épistémologique en impose la lecture à tous les spécialistes de l'histoire des religions. On en retiendra spécialement que, de même que l'on construit les comparables, on construit aussi la comparaison dans un champ de possibles. Car c'est 
une opération intellectuelle qui travaille sur les relations ou les facettes des objets pris en considération et qui a pour objectif d'apporter une plus-value de connaissance et d'intelligibilité des objets, et non pas d'en déterminer l'identité ou l'unicité, moins encore la supériorité.

\section{AUTEURS}

\section{CORINNE BONNET}

Université de Toulouse II-Le Mirail

corinne.bonnet@sfr.fr 